\title{
Wealth distribution in a network with correlations between links and success
}

\author{
S. Risau Gusman ${ }^{1}$, M. F. Laguna ${ }^{2}$ and J. R. Iglesias ${ }^{3}$ \\ 1 Instituto de Física, Balseiro, Centro Atómico Bariloche, 8400 - S. C. de \\ Bariloche, Argentina, srisau@cab.cnea.gov.ar \\ 2 The Abdus Salam International Center for Theoretical Physics. Strada Costiera \\ 11, 34014 - Trieste, Italy, mlaguna@ictp.trieste.it \\ 3 Instituto de Física, UFRGS, Caixa Postal 15051, 91501-970 - Porto Alegre, \\ Brazil, iglesias@if.ufrgs.br
}

\section{Introduction}

A study of the distribution of the income of workers, companies and countries was presented, a little more than a century ago, by Italian economist Vilfredo Pareto. He investigated data of personal income for different European countries and found a power law distribution that seems not to be dependent on the different economic conditions of the countries. In his book Cours d'Economie Politique [1] he asserted that in all countries and times the distribution of income and wealth follows a power law behaviour where the cumulative probability $P(w)$ of people whose income is at least $w$ is given by $P(w) \propto w^{-\alpha}$, where the exponent $\alpha$ is named today Pareto index, while the power law is known as Pareto law. The exponent $\alpha$ for several countries was $1.2 \leq \alpha \leq 1.9$. However, recent data indicates that, even though Pareto's distribution provides a good fit to the distribution of high range of income, it does not agree with observed data over the middle and low range of income. For instance, data from Japan [2, 3], Italy [4], India [5], Brazil [6], the United States of America and the United Kingdom [7, 8, 9] are fitted by a lognormal or Gibbs distribution with a maximum in middle range plus a power law for high income one.

The existence of these two regimes may be justified in a qualitative way by stating that in the low and middle income class the process of accumulation of wealth is additive, causing a Gaussian-like distribution, while in the high income class the wealth grows in a multiplicative way, generating the power law tail [3]. However, it is not clear if the difference between this two-regime behaviour and the original Pareto law is the results of an historical change of the income profile during the last century, or a characteristic of the data analysed. 
Different models of capital exchange among economic agents have been proposed trying to obtain the power law distribution for the wealthiest strata. Most of these models consider an ensemble of interacting economic agents that exchange a fixed or random amount of a quantity called "wealth". This wealth parameter represents the welfare of the agents. The exact choice of this parameter is not straightforward. For instance, when thinking of countries in the world economy, the GNP (Gross National Product) or some function of macroeconomic indicators could be a reasonable choice. In the case of companies, equity, share price or some suitable combination of them with outstanding debt are reasonable candidates. In the model of Dragulescu and Yakovenko [7] this parameter is associated with the amount of money a person has available to exchange. Within this model the amount of money corresponds to a kind of economic "energy" that may be exchanged by the agents in a random way and the resulting wealth distribution is a Gibbs exponential distribution, as it would be expected. An exponential distribution as a function of the square of the wealth is also obtained in a model where some action is taken, at each time step, on the poorest agent, trying to improve its economic state [10]. In the case of this last model a poverty line with finite wealth is also obtained, describing a way to diminish inequalities in a wealth distribution [11].

In order to try to obtain the power law tail several methods have been proposed. Keeping the constraint of wealth conservation a detailed studied proposition is that each agent saves a fraction - constant or random - of their resources $[13,14,15,16,19,20,12,17,18]$, fraction that introduces a multiplicative factor in the exchanges. One possible result of that model is condensation, i.e. the concentration of all the available wealth in just one or a few agents. To overcome this situation different rules of interaction have been applied, for example increasing the probability of favouring the poorer agent in a transaction $[19,18]$, or introducing a cut-off that separates interactions between agents below and above this cut-off [21]. Most of these models are able to obtain a power law regime for the high-income class, but for some values of the parameters, while for the low income, the regime can be approximately fit by an exponential or lognormal function. Finally it is worth quoting that Slanina [22] proposed a non-conservative version of the "gas" model[7], where the agents win or lose some extra wealth in the interaction, and he is able to obtain a power law regime for the high income class. One interesting point of this model is the non-conservation of wealth (or money) that makes it more realistic; on the other hand, the model is deterministic, not stochastic.

Here we would like to address the point that in all those models possible correlations between wealth and probability of interaction are not considered. That means that there are no correlations between the wealth of the agents and the probability of interaction between them. This seems to be at odds with the idea that people tend to strongly interact mainly with others of their own social and economic class[21] and also the fact that success in business is awarded with more business. One example are the internet based e-shops that 
are beginning to substitute traditional shops. When presented with different choices, people prefer to buy in e-shops that have better "references", i.e. in the shops that have made transactions with more customers. In that way successful traders are rewarded with more links. Another example: Inaoka et al [23] analyse the exchanges in Japanese banks, concluding that the bigger ones have more interactions between them and with the others than the small banks. The resulting network of interactions is very different for big banks (almost fully connected) than for small ones (a kind of star-like network).

Recently we have presented a model including correlations between wealth and the possibility of having an exchange[24]. In this model agents can trade just if they belong to the same economic class (i.e. their wealth difference is within a given range $u$ ) and the result is an extreme class polarization with the decline of the middle class. Here we present a different approach, correlating the success of an agent in their economics exchanges with its degree of connectivity. A model is considered where each agent possess a given amount of wealth, randomly chosen between the arbitrary values of 0 and $w_{\max }$. A different level of a randomly distributed risk aversion parameter is also attributed to each agent, as in previously discussed models, being this individual risk aversion level constant during the simulation. The agents are initially placed on a random lattice, with a given average connectivity $\bar{\nu}$. When the exchange of wealth between agents take place, every time an agent increase its wealth, it also increases its connectivity, that is, the number of neighbours that are linked to it. In the next section we describe the details of the model and the simulations, and in the last section we present the results and conclusions.

\section{Dynamic network model}

We consider a set of economic agents characterized by two parameters: a wealth $w_{i}$ and a risk aversion factor $\beta(i)$, with $0 \leq \beta(i) \leq 1$. The last parameter remains fixed during the whole process, and allows us to define the quantity $[1-\beta(i)]$ as the percentage of wealth that agent $i$ is disposed to risk. Agents are the nodes of a random network (i.e. a network having a Poisson distribution of connectivities) with average connectivity $\bar{\nu}$ and interactions are only allowed between connected agents.

The dynamics of the system consist first in choosing at random two agents connected by a link, which will exchange resources. Then, we put them to interact with the following rules: we establish that no agent can win more than the amount he puts at risk. This means that the amount that will be exchanged is the minimum value of the available resources of both agents, $d w=\min \left[\left(1-\beta_{1}\right) w_{1} ;\left(1-\beta_{2}\right) w_{2}\right]$.Finally, we introduce a probability $p \geq 0.5$ of favoring the poorer of the two partners, because increasing the probability of favoring the poorer agent is a way to simulate the action of the state or of some type of regulatory policy that tries to redistribute the resources $[17,11]$. Also, several authors $[13,14,12,18]$ have shown that without this prescription 
the system condensates, i.e. just one or a few agents concentrate the total wealth of the system. Here we determine this probability using a formula proposed by Scafetta et. al. [19, 20, 18],

$$
p=\frac{1}{2}+f \times \frac{\left|w_{1}-w_{2}\right|}{w_{1}+w_{2}}
$$

being $w_{1}$ and $w_{2}$ the respective wealths of the two partners in the exchange; $f$ is a factor going from 0 (equal probability for all agents) to $1 / 2$. Thus, in each exchange the poorer agent has probability $p$ of receiving the quantity $d w$ whereas the richer one has probability $1-p$.

Moreover, at the same time that the winner in the exchange increases his wealth by $d w$, he is also rewarded with a given number of links, proportional to the amount $d w$. These additional links could come from the loser agent (version $A$ of the model), or could be taken at random from any point of the lattice (version $B$ ).

We performed numerical simulation with these rules and found that, after a transient, the system arrives to a stationary state where the wealth has been distributed but also the network has changed from a random one to a web where the richer agents concentrate most of the links. This represents a society where the more successful agents obtain also better trade conditions, thus improving the opportunities of making more money. On the other side the situation will be not so unfair as expected for the poorer strata of the population. The smaller connectivity creates a kind of "protective screening" for the less favored agents, preventing them from losing more money.

\section{Results and Conclusions}

We consider a number of agents $N$ ranging from 5000 to 10000 and a number of average exchanges big enough to guarantee a stationary state $\left(10^{3}\right.$ to $10^{4}$ exchanges per agent). The initial wealth for each agent is chosen at random from an uniform distribution where between 0 and $w_{\max }$, being here $w_{\max }=$ 500. We investigate several values for the average number of links per agent, going from 5 to 80 links per agent in the case $N=5 \times 10^{5}$ agents. The initial distribution of links is a Poisson distribution.

In order to update the lattice at each exchange, we divide the total wealth of the system by the total number of links, attributing a "monetary" value to each link. The winner in a transaction also wins the equivalent number of links, rounded by elimination of any fractionary number. Finally the value of $f$ used to determine the probability $p$ of favoring the poorer agent has been set equal to $0 ., 0.1,0.3$ and 0.5 .

We show here three different cases: the static lattice, in order to have a reference for comparison, the case $A$ - where after the exchange the winner takes links from the loser up to a limit of leaving the loser connected by at least one link -, and the case $B$ - where the winner takes a link at random 
from any agent -. Notice that in all three cases the total number of links remains constant throughout the evolution.
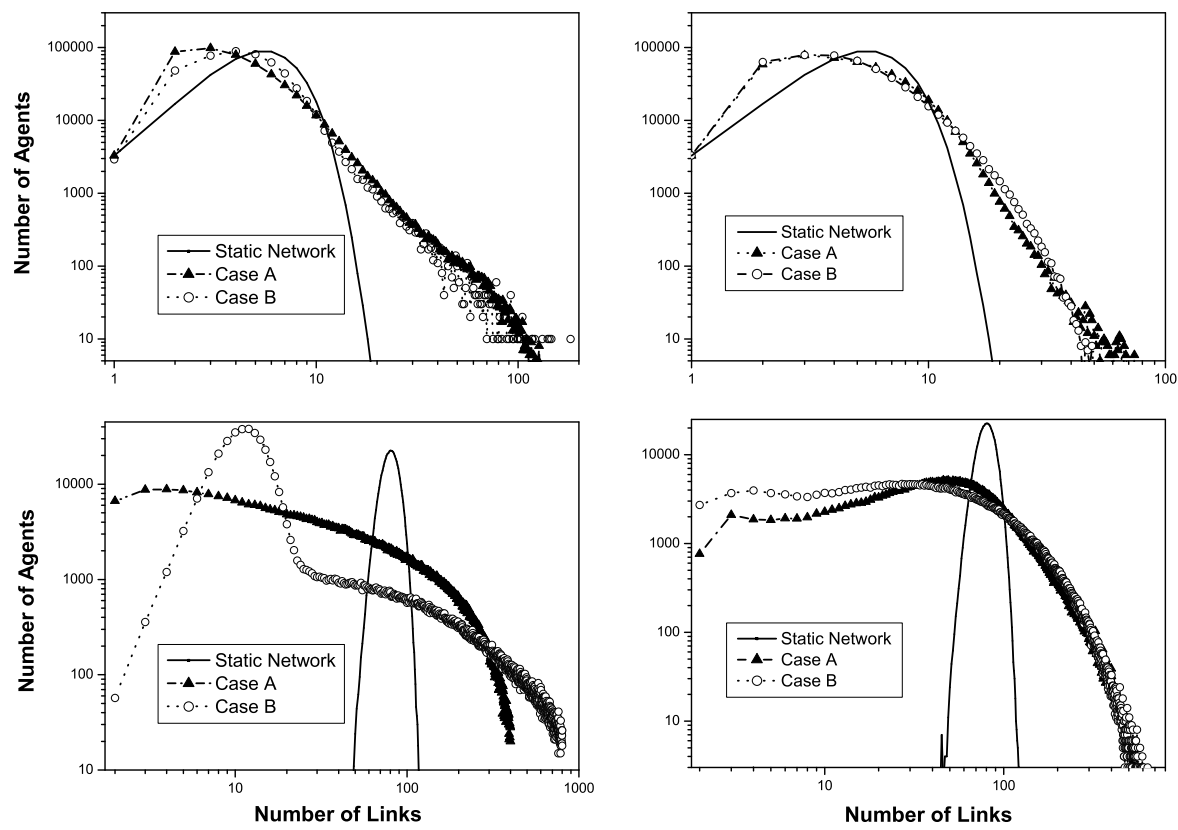

Fig. 1. Cumulative histogram of the asymptotic link distribution for $f=0.1$ (left column) and $f=0.5$ (right column), $\bar{\nu}=5$ (first row) and $\bar{\nu}=80$ (second row). The results are taken from 100 runs. The lines joining the symbols are only guides to the eye.

In Fig. 1 we show the asymptotic distributions of links for $f=0.1$ and $f=0.5$ (the poorer agent is maximally favored at each transaction) and for $\bar{\nu}=5$ and $\bar{\nu}=80$. In all cases the full curve corresponds to the initial distribution, that is also the static one, as the latter is not modified by the dynamics. It can be seen that in all cases the resulting distribution deviates significantly from the initial one: a few agents end up having a number of links much higher than the average, whereas most of the population has very few links. The maximum is always shifted to the left. This effect is most dramatic in the case $f=0.1, \bar{\nu}=80$ : for case $B$ the maximum is shifted from 80 links to only 10 , whereas some agents are connected to up to $\sim 800$ other agents. In the case $A$, for these same parameters, the resulting distribution is rather different: the maximum is much less pronounced but has been shifted to very low values $\nu \approx 1$, while the maximal number of links is also much smaller. Finally, in the case $f=0.5$, the effect of favoring the poorer agents seems to smooth out almost completely the differences between the dynamics A and B. 
The most interesting results concern the asymptotic wealth distribution. In Fig. 2 we present results for $f=0.1$ and $f=0.5, \bar{\nu}=5$ and $\bar{\nu}=80$. For $f=0.1$, (but also for small values of $f$ ), there appears a very high peak for low values of income: about 60 per cent of the agents own about one tenth of the average wealth. On the other hand, most of the wealth is owned by a few very rich agents. The personal wealth of these agents is about ten times greater than the average wealth. The differences between the different cases concern mainly the number of people in the middle class, loosely defined as the wealth interval between $w_{\max } / 10$ and $w_{\max }$, and the number of people in the upper class $\left(w>w_{\max }\right)$. One striking feature observed for $f=0.1$ is that in the high class the asymptotic distribution for case $\mathrm{A}$ follows a power law, whose exponent is $\sim-2$ (corresponding to a Pareto exponent -1 ). Also, it is rather surprising that the distribution for case $\mathrm{A}$ and the static one are almost identical for $f=0.1, \bar{\nu}=80$, even though the underlying lattices are very different (see Fig. 1).
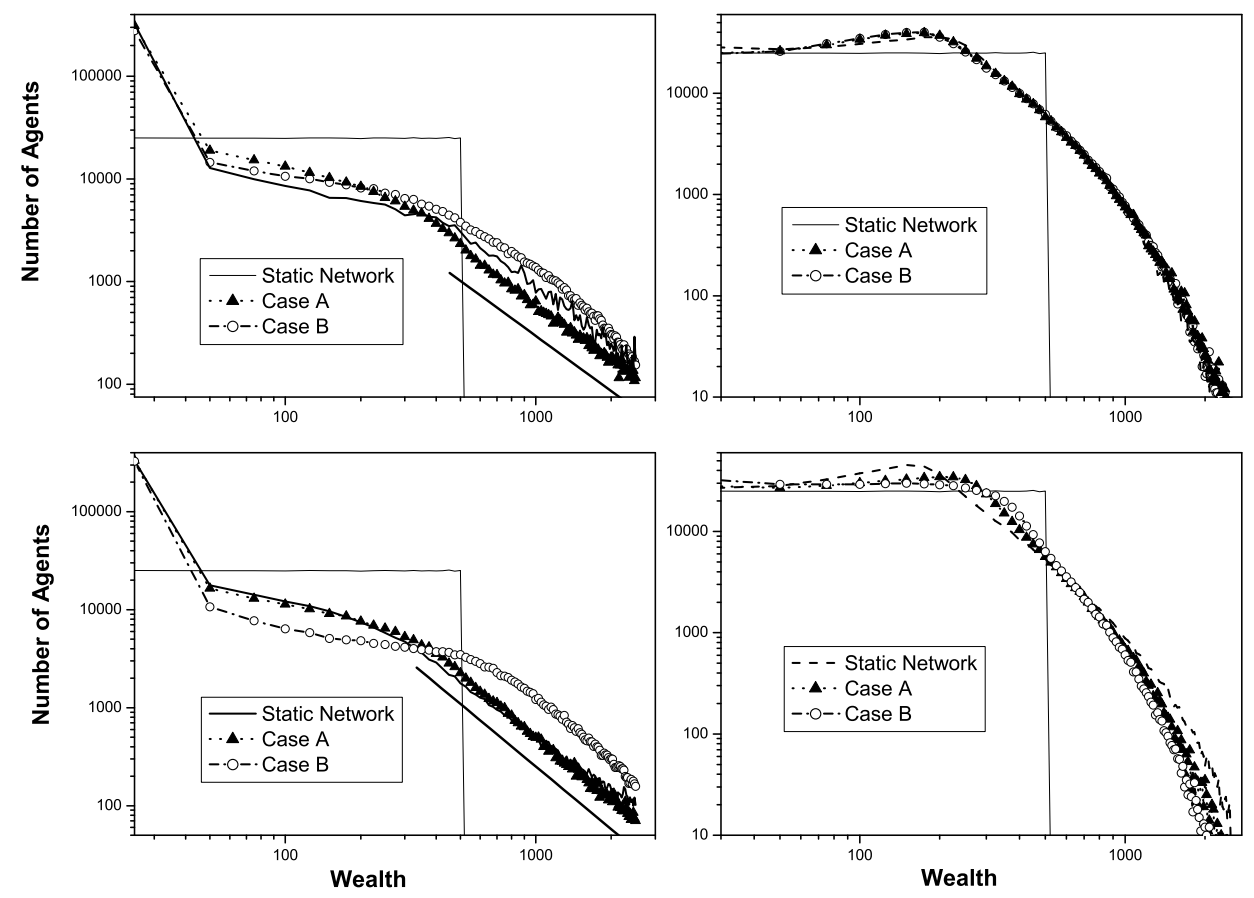

Fig. 2. Cumulative histogram of the asymptotic wealth distribution for $f=0.1$ (left column) and $f=0.5$ (right column), $\bar{\nu}=5$ (first row) and $\bar{\nu}=80$ (second row). The results are taken from 100 runs. For $\mathrm{f}=0.1$ the straight lines correspond to fits with a power law, whose exponents are -1.8 . The lines joining the symbols are only guides to the eye. 
As it has been observed for the links distribution, the differences between the three cases are smoothed out for high values of $f$. But the wealth distribution depends on the dynamics of the network for low values of $f$. It seems that the effect of the dynamics of links is important when there are no regulations in the exchanges $f \approx 0$. , and the result is that the number of agents in the middle class decreases while the number of agents in the very low or in the high income class increases, but the effect is not as pronounced as in ref. [24]. In the case of $f=0.5$ the wealth distribution looks similar to that of developed countries like Japan[2] or England [9]: A maximum in the distribution is observed for a "middle class" and for high income a power law may be drawn, but on a relatively narrow strip of wealth. The income of that "middle class" is almost the same average initial value of the wealth, while the number of very rich people is smaller by a factor of 10 compared to what happens for $f=0.1$.

In order to compare the different distributions between them and with empirical data, it is useful to determine the values of the Gini coefficients. As it can be observed in Table 1 differences among the different cases are only significant for low values of $f$, but in these cases the Gini coefficients are very far from being realistic. It is only for high values of $f$ that we obtain Gini indexes that are close to those observed in real societies. For $f=0$. and $f=0.1$ unfairness clearly increases with connectivity in case A and for the same parameters it also increases when switching from the static case to both dynamic lattices. On the other hand, for higher values of $f(f=0.3$ and $f=0.5)$, it seems that the reconnection of the lattice induces a kind of protective screening of the lower classes, being the Gini exponents slightly lower for both dynamic networks than for the static one (with the exception of case $A, \bar{\nu}=5$ ). Moreover, the Gini indices are even lower for case $B$, when the links are cut at random, than for case $A$, when they are taken from the loser agent. However, in all cases the changes are small, meaning that the reconnection of the lattice has little effect on inequalities.

\begin{tabular}{|c||c|c|c||c|c|c||c|c|c||}
\hline \multicolumn{1}{|c||}{} & \multicolumn{3}{c||}{ Static } & \multicolumn{3}{c||}{ Case A } & \multicolumn{3}{c||}{ Case B } \\
\cline { 2 - 10 } & 5 & 20 & 80 & 5 & 20 & 80 & 5 & 20 & 80 \\
\hline 0 & 0.816 & 0.9213 & 0.955 & 0.964 & 0.981 & 0.983 & 0.980 & 0.987 & 0.985 \\
\hline 0.1 & 0.793 & 0.878 & 0.91 & 0.884 & 0.897 & 0.915 & 0.89 & 0.868 & 0.873 \\
\hline 0.3 & 0.609 & 0.651 & 0.666 & 0.62 & 0.622 & 0.623 & 0.603 & 0.59 & 0.593 \\
\hline 0.5 & 0.443 & 0.466 & 0.473 & 0.441 & 0.432 & 0.428 & 0.433 & 0.422 & 0.424 \\
\hline
\end{tabular}

Table 1. Gini coefficients for the three dynamics treated in the article. The columns in each case correspond to the different values of $\bar{\nu}$ whereas the rows correspond to the different values of $f$.

We have also analyzed the correlation between the number of links of each agent and the wealth he has accumulated. For the static case we find that 
there is no correlation between connectivity and wealth, as it is expected i.e. we find that for all wealth classes the average connectivity coincides with its population average. For case $B$ we always find that there is a linear relationship: wealthier agents tend to be the more connected. On the other hand, in the case $A$ we find a clear linear relation only for high values of $f$. For small values of $f$ and not too large connectivity we find that the average connectivity is almost constant but, unlike the static case, this constant is smaller than that of the population average. There are, of course, agents with more links than the average and these are, surprisingly, very low income agents. Probably this is one of the reasons that for low values of $f$ the low income class has practically zero wealth: they have success in their exchanges but, as they can only get the same amount they risk, the average gain is negligible.
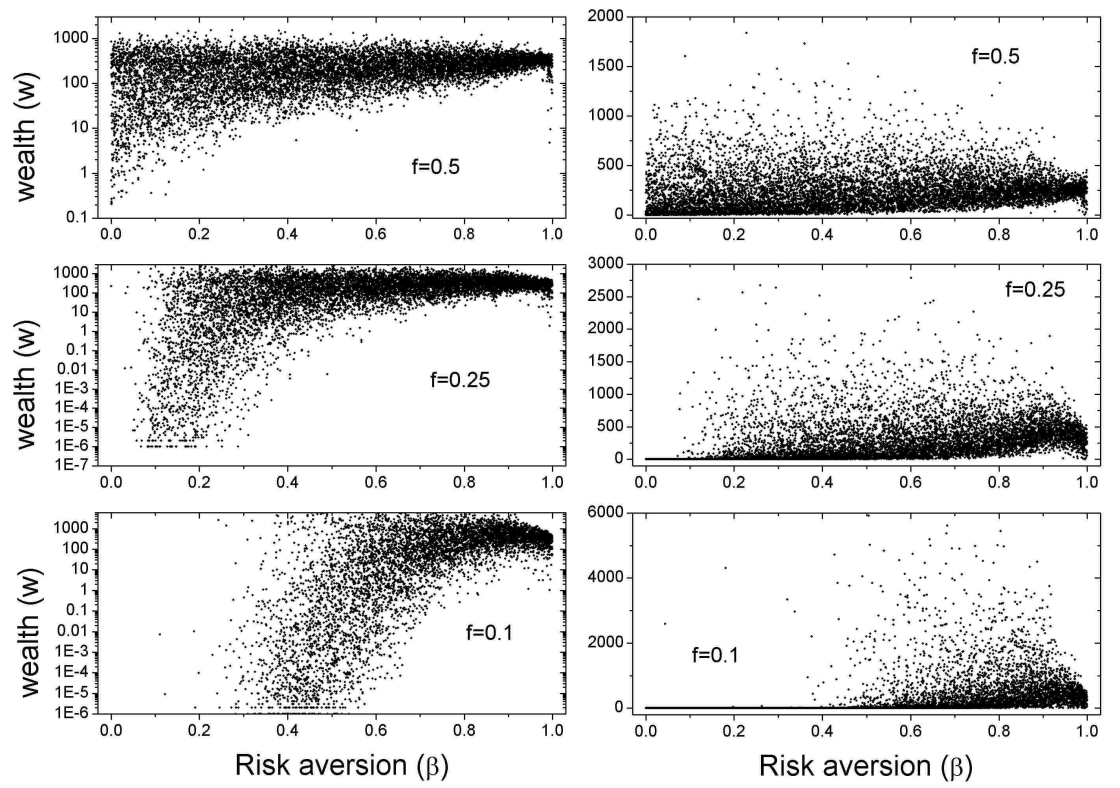

Fig. 3. Wealth vs. Risk aversion. Each point represents an individual, and the whole population has 10000 agents. The two columns only differ in the way the graphs are presented: a logarithmic ordinate scale in the left and normal ordinate in the right.

Finally, the correlation between wealth and risk aversion also presents some interesting features. In Fig. 3 we show some results for case $B$ (no noticeable differences are observed for different cases and connectivities)(.) For low values of $f$, only the most risk averting individuals have a significant chance to get rich. But, to get rich, $\beta$ should be of the order of 0.6 to 0.8 , as bigger values of $\beta$ imply very low sums put at stake. For high values of $f$ the situation changes to become more uniform; even very risk-prone individuals are able 
to get very wealthy. However, for high values of $f$ the wealthier individuals are significantly poorer than their counterparts for low values of $f$ : the richer agents have a wealth of the order of $4 \times w_{\max }$ for $f=0.5$ and $12 \times w_{\max }$ for $f=0.1$. Concerning the lower classes, it is possible to see on the logarithmic representation that a risk aversion of the order of 0.5 or higher guarantees a finite wealth of the order of $1 / 10 \times w_{\max }$ for $f=0.25$.

We conclude that the introduction of a correlation between connectivity and success in "commercial" exchanges produces a wealth distribution and Gini coefficients different than that of an static social lattice, but the effects are not as evident as expected. The more important parameter is still $f$, the probability of favoring the poorer agent in each exchange, and, in a minor degree, the average connectivity of the lattice. For low values of $f$ the wealth distribution is very unfair, still worse than in real societies. A finite fraction of the order of $2 / 3$ of the population has almost zero wealth while for the richer classes one obtains a rather robust power law. For high values of $f$ the distribution presents a maximum for finite values of the wealth, of the order of the average wealth, but there is still a finite fraction of agents with almost zero wealth. The effect of the average connectivity is also more evident for low values of $f$ but in all cases it seems that increasing the average connectivity is the best way to obtain power laws in the different situations studied. The effect of a dynamical network can be boosted either considering a correlation between the wealth of the agents, as in ref. [24], or modifying the rule that no agent can win more than the amount he puts at stake, because this strong constraint limits social and economical mobility.

\section{References}

1. Pareto V (1897), Cours d'Economie Politique, Vol. 2, F. Pichou, Lausanne

2. Aoyama H, Souma W and Fujiwara Y (2003), Growth and fluctuations of personal and company's income, Physica A: Statistical Mechanics and its Applications 324:352-358

3. Nirei M and Souma W (2004) Two factor model of income distribution dynamics, Santa Fe Institute (USA) report sfi0410029

4. Clementi F and Gallegati M (2005) Power law tails in the Italian personal income distribution, Physica A: Statistical and Theoretical Physics 350:427-438

5. Sinha S (2005) Evidence for Power-law tail of the Wealth Distribution in India, to appear in Physica A and condmat/0502166v1

6. Brasil (2004) http://www.ibge.gov.br/home/estatistica/populacao/trabalhoerendimento /pnad99/sintese/grafico.shtm

7. Dragulescu A and Yakovenko VM (2000) Statistical Mechanics of Money, The European J. of Physics B 17:723-729

8. Dragulescu A and Yakovenko VM (2001) Evidence for the exponential distribution of income in the USA, The European J. of Physics B 20:585-589

9. Dragulescu A and Yakovenko VM (2001) Exponential and power-law probability distributions of wealth and income in the United Kingdom and the United States, Physica A: Statistical and Theoretical Physics 299:213-221 
10. Pianegonda S, Iglesias JR, Abramson G and Vega JL (2003) Wealth redistribution with conservative exchanges Physica A: Statistical and Theoretical Physics 322:667-675

11. Pianegonda S and Iglesias JR (2004) Inequalities of wealth distribution in a conservative economy , Physica A: Statistical and Theoretical Physics 42:193199

12. Sinha S (2003) Stochastic Maps, Wealth Distribution in Random Asset Exchange Models and the Marginal Utility of Relative Wealth, Physica Scripta T106:59-64

13. Chatterjee A, Chakrabarti BK and Manna SS (2004), Pareto Law in a Kinetic Model of Market with Random Saving Propensity, Physica A: Statistical and Theoretical Physics 335:155-163

14. Chakrabarti BK and Chatterjee A (2004), Ideal Gas-Like Distributions in Economics: Effects of Saving Propensity, in "Applications of Econophysics", Ed. H. Takayasu, Conference proceedings of Second Nikkei Symposium on Econophysics, Tokyo, Japan, 2002, by Springer-Verlag, Tokyo. Pages 280-285. Preprint available: cond-mat/0302147.

15. Chakraborti A and Charkrabarti BK (2000) Statistical mechanics of money: how saving propensity affects its distribution, The European J. of Physics B $17: 167-170$

16. Patriarca M, Chakraborti A and Kaski K (2004) Statistical model with a standard Gamma distribution, Phys. Rev. E 70:016104

17. Iglesias JR, Gonçalves S, Pianegonda S, Vega JL and Abramson G (2003) Wealth redistribution in our small world, Physica A: Statistical and Theoretical Physics $327: 12-17$

18. Iglesias JR, Gonçalves S, Abramson G and Vega JL (2004) Correlation between risk aversion and wealth distribution, Physica A: Statistical and Theoretical Physics 342:186-192

19. Scafetta N, Picozzi S and West BJ (2002) Pareto's law: a model of human sharing and creativity cond-mat/0209373v1

20. Scafetta N, West BJ and Picozzi S (2003) cond-mat/0209373v1(2002) and A Trade-Investment Model for Distribution of Wealth cond-mat/0306579v2.

21. Das A and Yarlagadda S (2005) An analytic treatment of the Gibbs-Pareto behavior in wealth distribution cond-mat 0409329v1 and to be published in Physica A.

22. Slanina F (2004) Inelastically scattering particles and wealth distribution in an open economy, Phys. Rev. E 69:046102

23. H. Inaoka, H. Takayasu, T. Shimizu, T. Ninomiya and K. Taniguchi Physica A, 339(2004), 621.

24. Laguna MF, Risau Gusman S and Iglesias JR (2005) Economic exchanges in a stratified society: End of the middle class?, to appear in Physica A 